\title{
DESIGN AND TESTING OF UNBALANCED MASS MECHANICAL VIBRATION EXCITER
}

\author{
Nitinkumar Anekar ${ }^{1}$, V.V. Ruiwale ${ }^{2}$, Shrikant Nimbalkar ${ }^{3}$, Pramod Rao ${ }^{4}$ \\ ${ }^{1}$ Assistant Professor, Mechanical Department, MIT College of Engg, Pune, Maharashtra, India \\ ${ }^{2}$ Assistant Professor, Mechanical Department, MIT College of Engg, Pune, Maharashtra, India \\ ${ }^{3}$ Assistant Professor, Mechanical Department, MIT College of Engg, Pune, Maharashtra, India \\ ${ }^{4} U G$ Student, Mechanical Department, MIT College of Engg, Pune, Maharashtra, India
}

\begin{abstract}
A vibration exciter is a machine which produces mechanical vibratory motion to which the test object is subjected. This article presents design, construction, performance and testing of mechanical vibrations exciter, which have unbalanced mass to generate uniaxial vibrations. The exciter is designed to produce a given range of harmonic or time dependent excitation force and displacement through a given range of frequencies. The mechanical vibration exciter produces vibrations due to centrifugal force of rotating eccentric mass. The vibrations produced lie in the low frequency range. The construction of working device and its important parts are described here. The most important part of exciter is unbalanced mass attached with rotating disc of motor. Exciter has unbalanced mass at one end of disc, base frame, top plate as platform, springs and motor. This exciter is used for testing of welded parts, consolidation of concrete, concrete filling in mould. The obtained experimental results are in line with theoretical results.
\end{abstract}

Keywords: Exciters, unbalanced mass, steady state vibration, amplitude, frequency ratio, tests.

\section{INTRODUCTION}

A vibration exciter is a machine which produces mechanical vibratory motion to test object. The exciters are designed to produce a given range of harmonic or time dependent excitation force and displacement through a given range of frequencies. These machines can be mechanical, electrohydraulic or electro-dynamic in nature. The vibration exciters available in market were too costly for small scale applications so there was a need of for relatively low cost exciter which can be used for low frequency range. This can be used for experimentation purpose and testing product at different frequencies.

Certain machines and structures that develop excessive vibrations during their life and it may be required to make a diagnostic vibration analysis to prevent an impending failure of some of components. Such components can be tested using vibration exciters. Different types of vibration exciters are used for development, simulation, production, studying the effects of vibration and for evaluating physical properties of materials or structures. This paper also provides a brief description about different types of vibration exciters, its advantages and disadvantages over mechanical vibration exciter. It includes theory on forced vibration exciters.

The mechanical vibration exciter is tested for different application i.e. specimen and consolidation of cement. The actual testing of machine is done by varying speed of motor and the corresponding amplitude is noted. Vibration exciters are used to produce cyclic excitation force at a required frequency. The cyclic excitation force produced by the exciter can be applied to the machine or structure to study its dynamic characteristics. The excitation force is usually a sinusoidal or random type signal applied with a number of discrete frequencies over a specific frequency range of interest.

A.M. Wahl [1] gives information about various types of springs. This information is used while designing spring by designers and engineers. S. S. Rao [2] gives information on types of vibration, vibration analysis of single degree of freedom and multi degree of freedom systems. The effects of viscous, coulomb and hysteretic damping are also discussed. Design Data Book [3] gives information about standard values of various parameters required in design procedure of springs, nut, bolts. Norton[4] gives basic information of designing of small components come in exciter like nut, bolt, spring, structure, selection of motor etc. Pluta J.[6] and Je-Hoon Kim [7] gives idea of design and experimentation of bi-axial exciter of mechanical vibrations as well as electromagnetic vibration exciter for the Rapping of an Electrostatic Precipitator. After survey we found that, vibration exciters are costly and it is not viable so their work inspires us to design and manufacture unbalanced mechanical vibration exciters for small scale applications and testing purpose.

\subsection{Types of Exciters}

The three types of vibration exciters are commonly used in several applications as: Electro-dynamic exciter, Hydraulic exciter and Mechanical exciter. Their features are as given in TABLE I [5]. Further mechanical vibration exciters are having following types: 

a. Eccentric and connecting link
b. Scotch yoke
c. Cam and follower
d. Rotating unbalance mass

Table- 1: Features of Exciters

\begin{tabular}{|l|l|l|l|}
\hline $\begin{array}{l}\text { Exciter Type } \\
\text { Parameters }\end{array}$ & Mechanical & $\begin{array}{l}\text { Electro- } \\
\text { dynamic }\end{array}$ & $\begin{array}{l}\text { Electro- } \\
\text { Hydraulic }\end{array}$ \\
\hline Frequency & $2-50 \mathrm{~Hz}$ & $2-10,000 \mathrm{~Hz}$ & $0.1-500 \mathrm{~Hz}$ \\
\hline $\begin{array}{l}\text { Maximum } \\
\text { Displacement }\end{array}$ & $2.5 \mathrm{~cm}$ & $2.5 \mathrm{~cm}$ & $50 \mathrm{~cm}$ \\
\hline $\begin{array}{l}\text { Maximum } \\
\text { Acceleration }\end{array}$ & $20 \mathrm{~g}$ & $100 \mathrm{~g}$ & $20 \mathrm{~g}$ \\
\hline $\begin{array}{l}\text { Maximum } \\
\text { Force }\end{array}$ & $4500 \mathrm{~N}$ & $2000 \mathrm{~N}$ & $450,000 \mathrm{~N}$ \\
\hline $\begin{array}{l}\text { Excitation } \\
\text { Waveform }\end{array}$ & $\begin{array}{l}\text { Sinusoidal } \\
\text { only }\end{array}$ & $\begin{array}{l}\text { High } \\
\text { flexibility } \\
\text { and } \\
\text { accuracy }\end{array}$ & $\begin{array}{l}\text { Average } \\
\text { flexibility }\end{array}$ \\
\hline
\end{tabular}

Let's see rotating unbalance mass type mechanical exciter. In this exciter, an eccentric mass is mounted on a rotating shaft to generate vibration. The product of the mass and the distance of its centre from the axis of rotation is referred to as the mass unbalance or the rotating unbalance or simply the unbalance. The force resulting from this unbalance is referred to as the unbalanced force. This type exciter consists of at least one rotating unbalance mass directly attached to the vibrating table. The table and rotating unbalance are supported from the base or frame by springs. The rotating unbalance generates an oscillating force which drives the table.

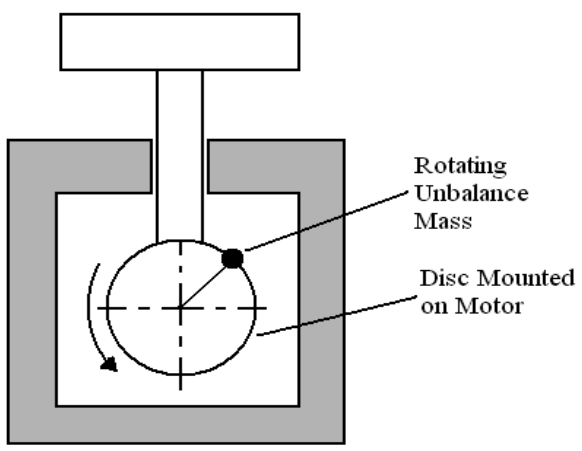

Fig-1: Rotating unbalance mass

The unbalance force is transmitted through bearings directly to the table mass, causing a vibratory motion without reaction of the force against the base. A vibration machine employing this principle is referred to as a reaction machine since the reaction to the unbalance force is supplied by the table itself rather than through a connection to the floor or ground. One or two rotating unbalanced masses produces vibratory motion in perpendicular or in parallel direction to the vibration table. The rotating unbalances turning in opposite directions are phased so that the unbalance forces add in the desired direction and cancel in other directions. The effective generated force from the two rotating unbalances is midway between the two axes of rotation and is normal to a line connecting the two it.

\subsection{Advantages, Disadvantages and Applications of}

\section{Mechanical Exciter}

The advantages, disadvantages and applications of mechanical exciter are given as below [5]:

\section{Advantages}

- $\quad$ The forces generated by the rotating unbalances are transmitted directly to the table without dependence upon a reactionary force against a heavy base or rigid ground connection.

- The cost of mechanical exciter is less compared to other exciters.

- $\quad$ There are no leakage problems as in hydraulic exciters.

- Defective electrical components and connections fail under the induced vibration. This exciter eliminates embarrassing and costly repair and difficult tracing of circuits in the field.

\section{Disadvantages}

- Mechanical exciter cannot be used in high temperature, humidity and altitude environments.

- It can only be used for small applications.

- The frequency range is small compared to hydraulic and pneumatic.

\section{Applications}

- It can be used for the purpose of studying the effects of vibration or for evaluating physical properties of materials or structures.

- $\quad$ It can also be used for finding the natural vibrating frequencies.

- $\quad$ It is used for testing of welded, soldered parts and consolidation of cement, concrete filling in mould

\section{NUMERICAL MODEL}

Here we used concept of forced vibration with mass excitation. The concept is given as below: We know that, a rotating machine such as: Centrifugal pumps, turbines, electric motor, etc, have some amount of unbalance left in them even after balancing with the help of precession balancing machines. This unbalance is a common source of forced vibrations. The unbalance in rotating machines is measured in terms of mass ' $\mathrm{m}$ ' rotating with its centre of gravity at a distance ' $\mathrm{e}$ ' from the axis of rotation. The centrifugal force due to unbalance mass ' $\mathrm{m}$ ' acts as harmonic excitation force. Consider a machine with a rotating unbalance supported on a spring and a damper (i.e. mounted on an elastic support), as shown in Fig.2.[2]

\section{Assumption}

It is assumed that the system is constrained to move vertically having single degree of freedom. Let,Total vertical displacement of mass of machine excluding unbalanced rotating mass $(M-m)=x$ 
Total vertical displacement of unbalanced rotating mass ' $m$ ' $=(x+e \sin \omega t)$

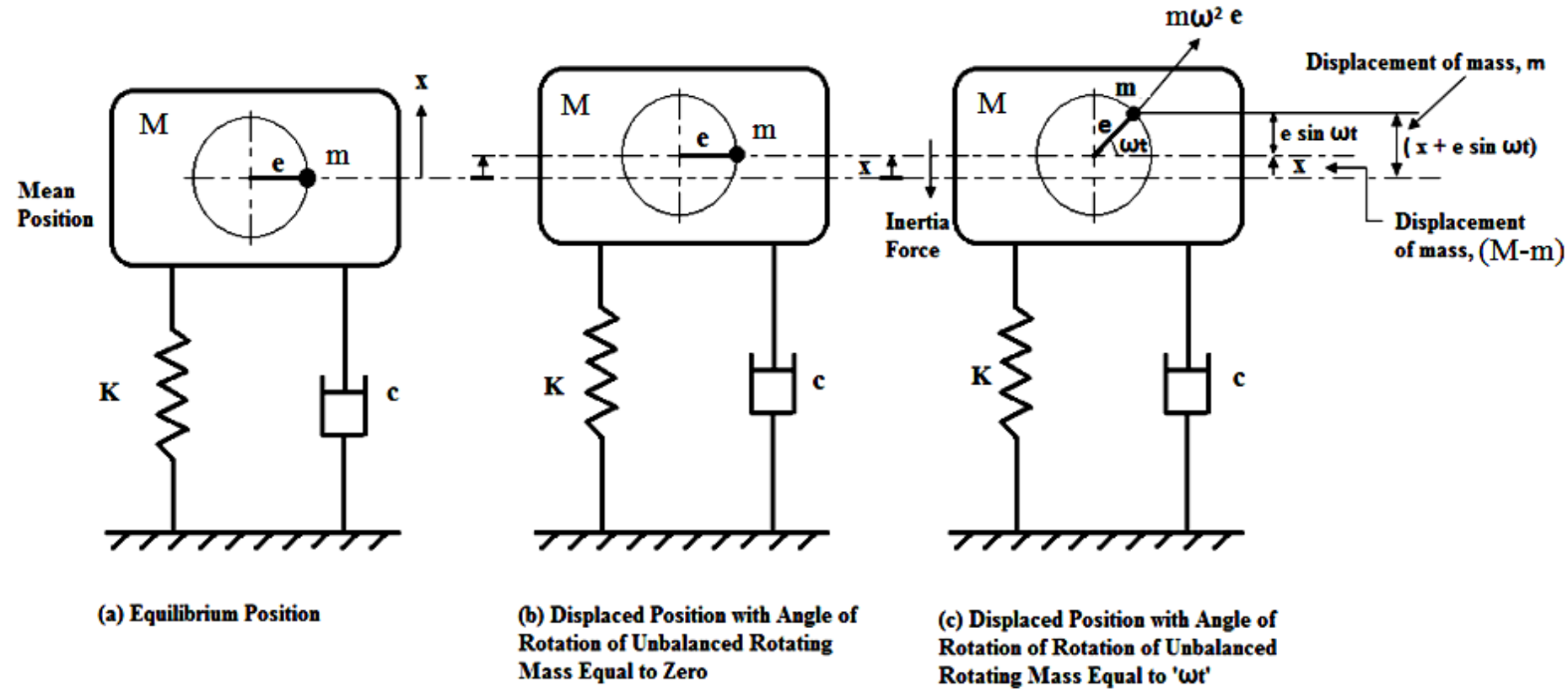

Fig -2: Rotating Unbalance

Inertia force due to mass of machine excluding unbalanced rotating mass $(\mathrm{M}-\mathrm{m})=(M-m) \ddot{x}$

Inertia force due to unbalanced rotating mass ' $m$ '

$=m \frac{d^{2}}{d t^{2}}(x+e \sin \omega t)$

$=m\left(-\omega^{2} e \sin \omega t\right)$

$=\left(m \omega^{2} e \sin \omega t\right)$

Spring force $=K x$

Damping force $=c x$

Consider F.B.D. of machine shown in Fig.3. below

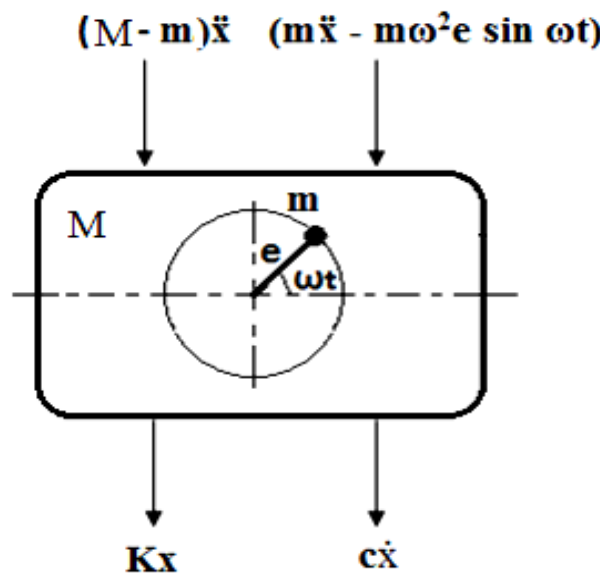

Fig.-3: Free Body Diagram of Machine

Use D’Alemnerts's principle, $\sum[$ Inertia force + External forces $]=0$

$$
\begin{aligned}
& (M-m) \ddot{x}+\left(m \ddot{x}-m \omega^{2} e \sin \omega t\right)+c \dot{x}+K x=0 \\
& M \ddot{x}+c \dot{x}+K x=m \omega^{2} e \sin \omega t
\end{aligned}
$$

This equation is the linear; second order differential equation, of motion for a forced damped vibrations due to rotating unbalance.

The complete solution for differential Equation is,

$$
X=X_{C}+X_{P}
$$

$$
X=X_{1} e^{-\xi \omega_{n} t} \sin \left(\sqrt{1-\xi^{2}} \omega_{n} t+\phi_{1}\right)+\frac{m e \omega^{2} \sin (\omega t-\phi)}{K \sqrt{\left(1-r^{2}\right)^{2}+(2 \xi r)^{2}}}
$$

Here $r=\frac{\omega}{\omega_{n}}$ i.e. frequency ratio

The amplitude of steady state vibrations is given as below:

$\frac{X}{\left(\frac{m e}{M}\right)}=\frac{r^{2}}{\sqrt{\left(1-r^{2}\right)^{2}+(2 \xi r)^{2}}}$

Here, $\frac{X}{\left(\frac{m e}{M}\right)}$ is dimensionless amplitude generated because of rotating unbalance in machine. 
The plot of dimensionless amplitude vs frequency ratio is given in Fig.4 [2] gives information, when the speed is zero; the dimensionless amplitude is also zero. Hence all curves start from origin. At resonance, the dimensionless amplitude is limited only by the damping present in the system. When the frequency ratio is very large, the dimensionless amplitude tends to unity.

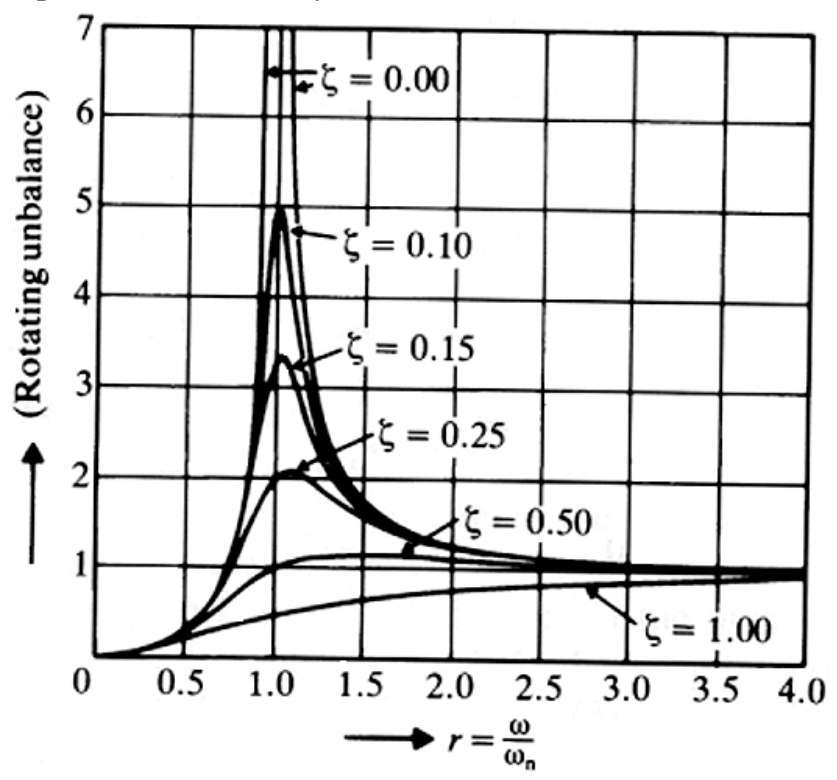

Fig -4: Plot of dimensionless amplitude Vs frquency Ratio

\section{DESIGN AND CONSTRUCTION}

The exciter machine was designed for experimentation purpose and testing products at different frequencies. The exciter is designed taking both static and dynamic load conditions [3,4]. Spring is important part in design so it is designed under static and dynamic conditions to check under surging and fatigue[1].We have designed a mechanical vibration exciter as shown in Fig.5. It shows a schematic diagram of exciter (Overall Dimensions: $500 \mathrm{~mm}$ x $500 \mathrm{~mm} \times 750 \mathrm{~mm}$ ). The equipment is capable of sustain excitation.

The exciter is consisting of five main parts:

$\begin{array}{cl}\text { i. } & \text { Base frame, } \\ \text { ii. } & \text { Drive system (motor), } \\ \text { iii. } & \text { Eccentric mass, } \\ \text { iv. } & \text { Spring } \\ \text { v. } & \text { Top plate. }\end{array}$

The exciter is mainly made of mild steel, except elements like disc attached to motor, which is made of aluminium. The base frame is made of channel. The drive system includes a permanent magnet type DC motor with variable speed. The disc with eccentric mass is attached to motor shaft at one end. This system is fixed to top plate which is also used to hold the samples. To achieve different excitations the variable speed knob is attached to DC motor to control both speed and excitations. The drive system achieves a speed between $0 \mathrm{rpm}$ to $1440 \mathrm{rpm}$.

The working of this exciter is as follows. First ensure the wiring connections. Connect the vibration exciter to single phase power supply via plug. Switch on the power supply.

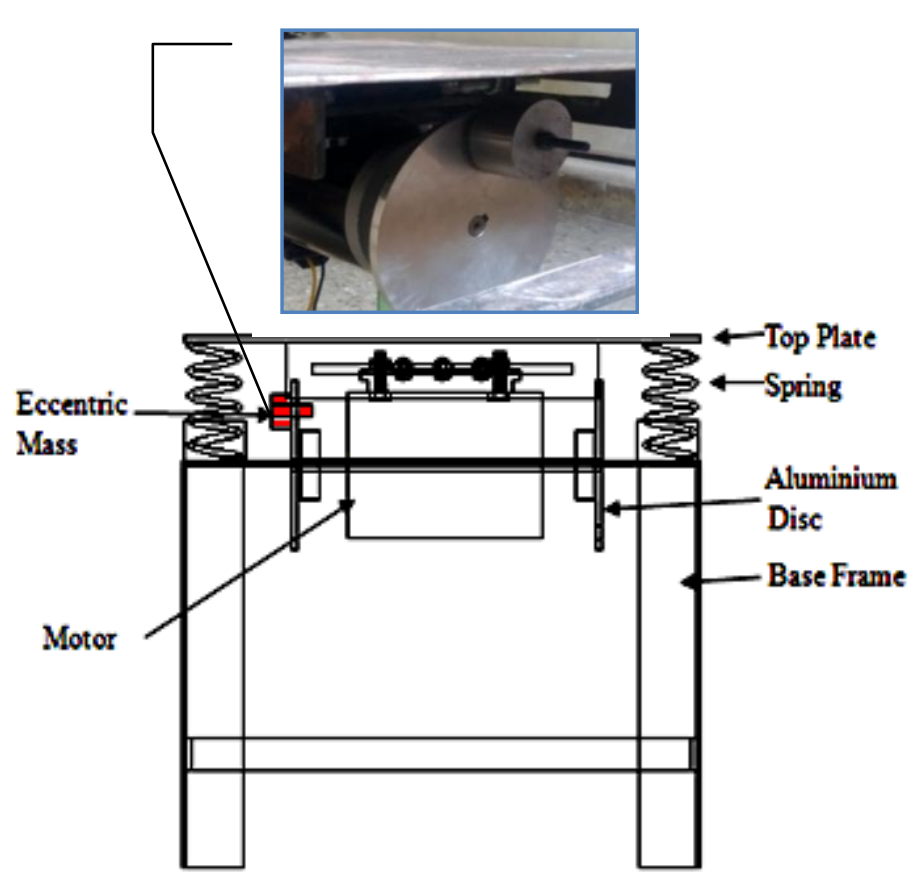

Fig.-5: Schematic Diagram of Mechanical Vibration Exciter

Slowly vary the speed of motor by using potentiometer. Note down the amplitude during different speed of motor. Slowly bring the speed of motor back to zero using potentiometer. Switch of the power supply.

\section{TESTING}

In order to determine the performance of the proposed exciter, the following tests are performed which we are discussed here. This exciter has a lot of applications. It is used in moulding, consolidation of cement, grain cleaning and many other purposes. Here we have given the procedure of concrete filling in the mould with the help of exciter as given below:

- Table should be bolted to the floor so as to avoid any loss of vibrations.

- $\quad$ Put the mould on to the table top. Then start to fill your mould with concrete slowly. Leaving the mould on the table shall then allow the air bubbles to escape by coming to the surface.

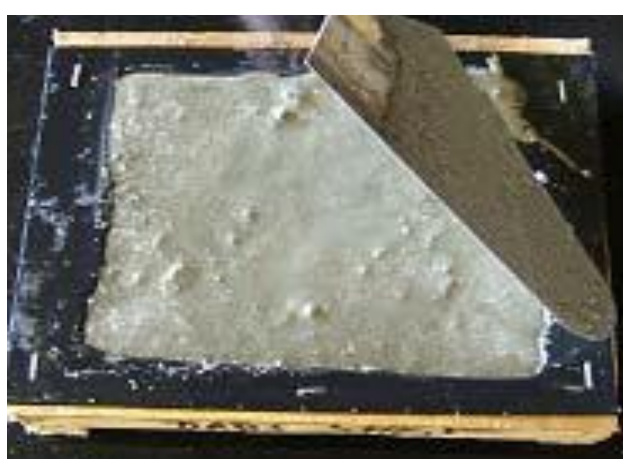

Fig.-6: Concrete filling in mould 


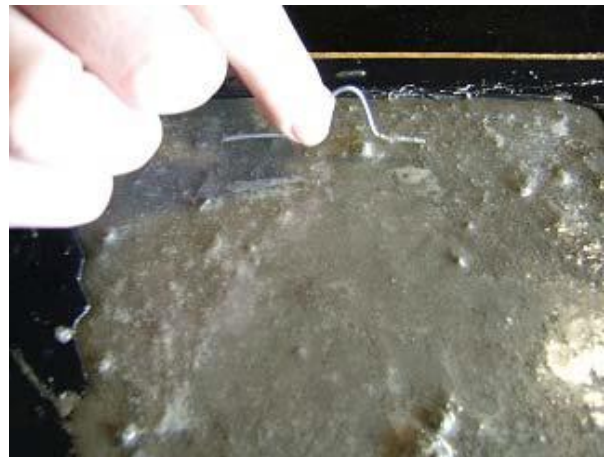

Fig.-7: Mixing additional pieces in mould like hooks

- Then level the mix off using a trowel as shown in Fig. 6. This gives a smooth and leveled base for a professional finish. The mould should then be left to set.

- $\quad$ The mould may need certain additional pieces such as hooks or tubing to complete the product. In the Fig.7, a wall plaque, a hook is placed into the mix after vibration has concluded.

- Mould material mixing is totally depends on wet or dry type of mixture. Guide rails can be clamped to the table top using pieces of timber to stop mould following off table during operation.

Similarly the exciter is used for consolidation of cement. The Fig. 8 and Fig. 9 gives the clear view of consolidation.

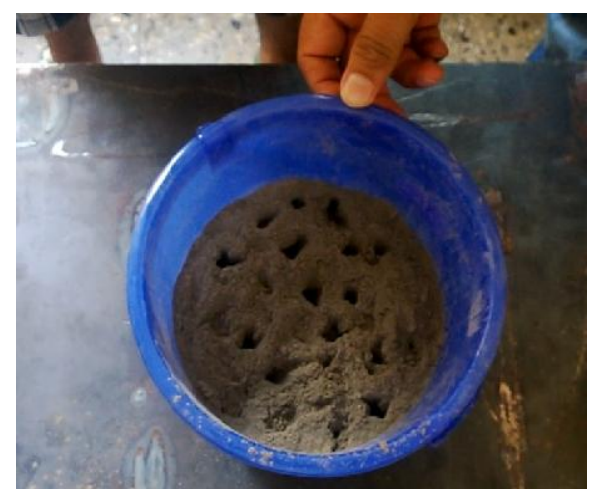

Fig.-8: Cement before Consolidation

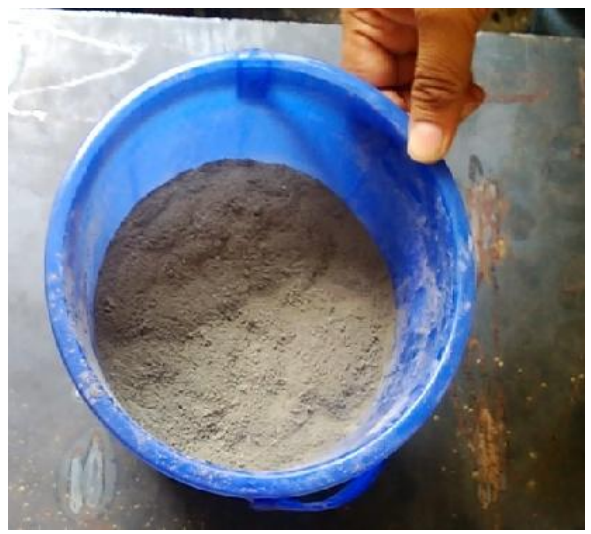

Fig.-9: Cement after Consolidation

\section{RESULTS AND DISCUSSION}

First find the damping co-efficient of the machine. The actual measurement of amplitude is done by varying the speed of motor. To calculate natural frequency and damping co-efficient for rotating unbalanced mass, the observations taken from machine are:

Unbalanced mass $=0.25 \mathrm{~kg}, 0.5 \mathrm{~kg}, 0.75 \mathrm{~kg}$,

Eccentric radius $=0.06 \mathrm{~m}$,

Mass of Table $=8.4 \mathrm{~kg}$,

Mass of guideways $=1.2 \mathrm{~kg}$,

Mass of studs $=0.4 \mathrm{~kg}$,

Mass of flange $=0.8 \mathrm{~kg}$,

Mass of disc $=0.5 \mathrm{~kg}$,

Total mass including unbalanced mass $=17.3 \mathrm{~kg}$.

Using equation (4), we get amplitude vs speed plots at unbalanced mass $0.25 \mathrm{~kg}, \quad 0.5 \mathrm{~kg}$ and $0.75 \mathrm{~kg}$ having eccentricity $60 \mathrm{~mm}$ as shown in Fig. 10 to12. From these figures we said that as speed increase, amplitude of vibration increase upto resonance speed and then decrease. Here, we clearly have seen that the experimental data matches with theoretical results. The plots shows us that as unbalanced mass increases, amplitude of vibration increases which is true as we compared with theory given in Fig. 4.

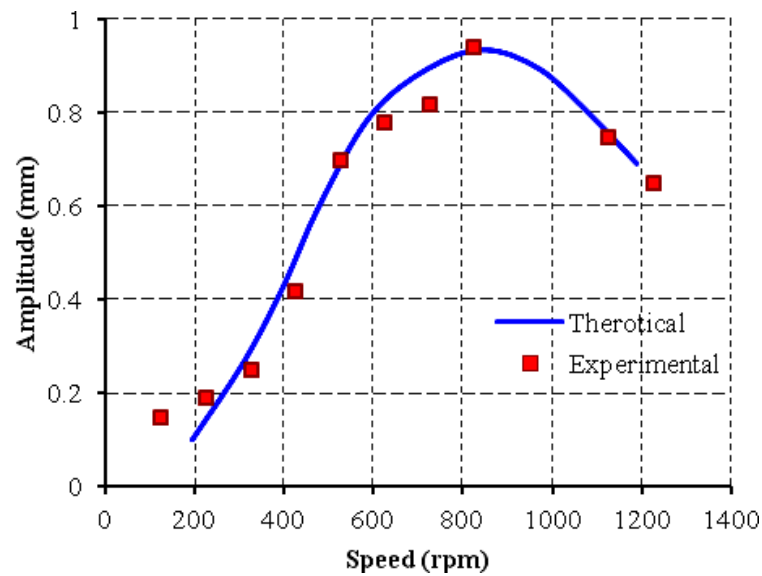

Fig.-10: Amplitude Vs Speed for unbalanced mass $0.25 \mathrm{~kg}$

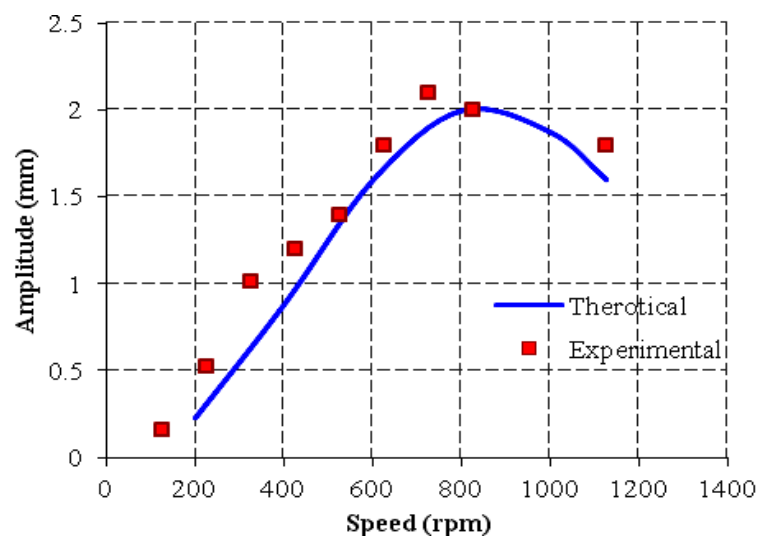

Fig.-11: Amplitude Vs Speed for unbalanced mass $0.5 \mathrm{~kg}$ 


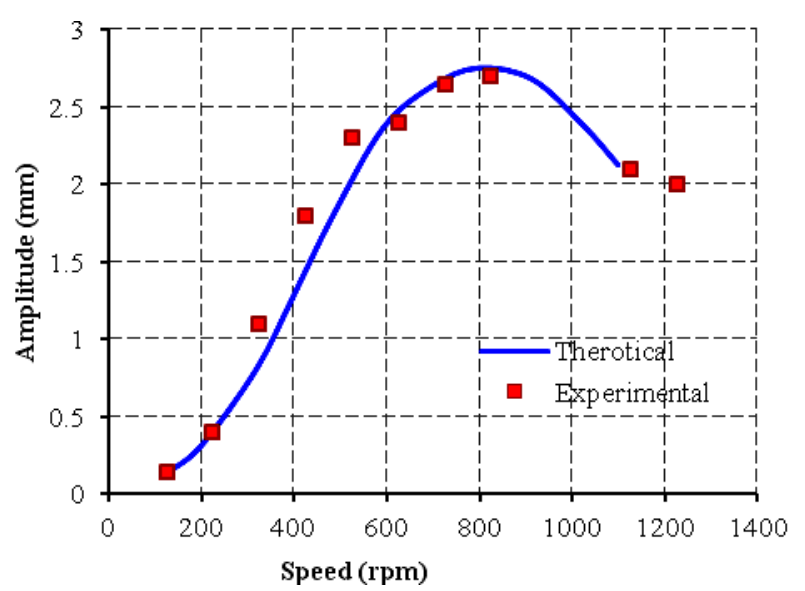

Fig.-12: Amplitude Vs Speed for unbalanced mass $0.75 \mathrm{~kg}$

The eccentricity of the unbalanced mass can be changed by providing holes at different radii on the disc. This can be done to achieve variable frequency. Here we have taken three different eccentricities as $20 \mathrm{~mm}, 40 \mathrm{~mm}$ and $60 \mathrm{~mm}$. Its results are given as in Fig.13. From this plot, we said that as eccentricity increase, the amplitude of vibration also increase upto resonance speed and then decrease.

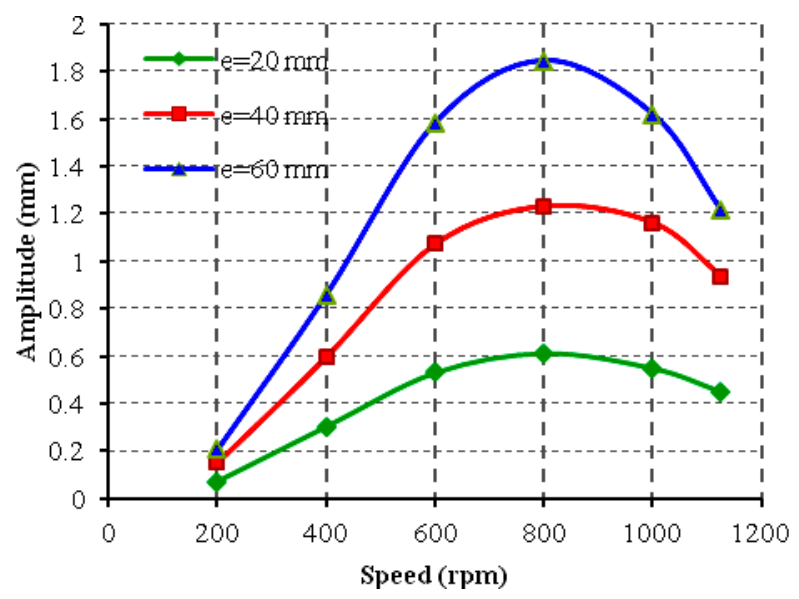

Fig.-13: Amplitude Vs Speed plot for eccentricity $20 \mathrm{~mm}, 40 \mathrm{~mm}, 60 \mathrm{~mm}$ at unbalanced mass $0.5 \mathrm{~kg}$

From these plots we said that our designed and manufactured unbalanced mass mechanical vibration exciter is good product.

\section{CONCLUSIONS}

Here the design, construction, performance and testing of an unbalanced mass mechanical vibration exciter were discussed. From observations we found that machine has internal stiffness and damping. The amplitude of vibration initially increases with increase in speed and reaches at maximum value (at resonance speed) and then decreases. We also found that raw cement can be satisfactorily consolidated at different speeds of vibration exciter. The resonance occurs at in the range of circular frequency of $750 \mathrm{rpm}$ and $850 \mathrm{rpm}$. Here we get different frequency response curve due to change in eccentric mass and change in their eccentricity. The results are in accordance with standard plots. Our testing machine permits to interchange different excitations according to the user requirements. An additional advantage of our testing machine is the lower cost and smaller size compared to other commercial vibration exciters. Future efforts will address the use of this equipment to check behaviour of cracks of components subjected to a particular frequency of vibrations and other applications.

\section{ACKNOWLEDGEMENTS}

The authors are grateful for the support MIT College of Engineering, Pune. The authors would also like to thanks Mr. Nikhil Saggam, Mr. Rohit Kumbhar and Mr. Ashish Sorathiya for their assistance in the work.

\section{NOMENCLATURE}

$M$ Total mass of machine including unbalanced mass, $(\mathrm{kg})$

$m$ Unbalanced mass $\mathrm{kg}$ )

$e$ Eccentricity of the unbalanced mass $(\mathrm{kg})$

$\omega$ Angular velocity of rotation of unbalanced mass, ( $\mathrm{rad} / \mathrm{s})$

$K$ Spring stiffness of the support $(\mathrm{N} / \mathrm{m})$

$c$ Damping coefficient $(\mathrm{N}-\mathrm{s} / \mathrm{m})$

$r$ Frequency ratio

$X$ Amplitude of vibration ( $\mathrm{mm}$ )

$\xi$ Damping factor

\section{REFERENCES}

[1] A. M. Wahl, "Mechanical Spring Design", McGrawHill, $2^{\text {nd }}$ Edition.

[2] S.S. Rao, "Mechanical Vibrations", Addison-Wesley, $4^{\text {th }}$ Edition.

[3] "PSG design data book", Central Book Publishers; $3^{\text {rd }}$ Edition

[4] Robert L. Norton, "Machine Design: An Integrated Approach," 2nd Ed., 2002.

[5] C. M. Harris and A.G. Piersol, "Harris' Shock and Vibration Handbook", McGraw-Hill, $5^{\text {th }}$ Edition

[6] Pluta J., Orkisz P., "Bi-axial exciter of mechanical vibrations", Carpathian Control Conference (ICCC), 2012 13th International , May 2012, pp. 568-572.

[7] Je-Hoon Kim, Jin-Ho Kim, Sang-Hyun Jeong, and Bang-Woo Han, "Design and Experiment of an Electromagnetic Vibration Exciter for the Rapping of an Electrostatic Precipitator", Journal of Magnetics 17(1), 2012,pp. 61-67. 